\title{
Existence and Multiplicity of Solutions for a Periodic Hill's Equation with Parametric Dependence and Singularities
}

\author{
Alberto Cabada ${ }^{\mathbf{1}}$ and José Ángel Cid ${ }^{\mathbf{2}}$ \\ ${ }^{1}$ Departamento de Análise Matemática, Facultade de Matemáticas, \\ Universidade de Santiago de Compostela, 15782 Santiago de Compostela, Spain \\ ${ }^{2}$ Departamento de Matemáticas, Universidad de Jaén, Campus Las Lagunillas, Ed. B3, 23071 Jaén, Spain
}

Correspondence should be addressed to Alberto Cabada, alberto.cabada@usc.es

Received 5 July 2010; Revised 27 January 2011; Accepted 24 February 2011

Academic Editor: Pavel Drábek

Copyright (C) 2011 A. Cabada and J. Á. Cid. This is an open access article distributed under the Creative Commons Attribution License, which permits unrestricted use, distribution, and reproduction in any medium, provided the original work is properly cited.

We deal with the existence and multiplicity of solutions for the periodic boundary value problem $x^{\prime \prime}(t)+a(t) x(t)=\lg (t) f(x)+c(t), x(0)=x(T), x^{\prime}(0)=x^{\prime}(T)$, where $\lambda$ is a positive parameter. The function $f:(0, \infty) \rightarrow(0, \infty)$ is allowed to be singular, and the related Green's function is nonnegative and can vanish at some points.

\section{Introduction and Preliminaries}

In the recent paper [1], the authors obtain existence, multiplicity, and nonexistence results for the periodic problem

$$
x^{\prime \prime}(t)-k^{2} x(t)=\lambda g(t) f(x), \quad x(0)=x(2 \pi), \quad x^{\prime}(0)=x^{\prime}(2 \pi),
$$

depending on the parameter $\lambda>0$. Although not explicitly mentioned in [1], we point out the important fact that the related Green's function of (1.1) is strictly negative for all $k>0$.

The aim of this paper is to give complementary results to those of [1] for the case of a nonnegative related Green's function. In particular, we will deal with problem

$$
x^{\prime \prime}(t)+a(t) x(t)=\lambda g(t) f(x)+c(t), \quad x(0)=x(T), \quad x^{\prime}(0)=x^{\prime}(T),
$$


assuming that its Green's function is nonnegative (for instance, if $a(t)=k^{2}$, this means $0<$ $k \leq \pi / T)$. Moreover, in order to give wider applicable results, we will also allow $f(x)$ to be singular at $x=0$ (the reader may have in mind the model $f(x)=1 / x^{\alpha}$, for some $\alpha>0$ ).

We note that analogous arguments have been developed in [2] for the fourth-order discrete equation

$$
u(k+4)+M u(k)=\lg (k) f(u(k))+c(k), \quad u(i)=u(T+i), i=0, \ldots, 3 .
$$

The main tool used in this paper is Krasnoselskii's fixed point theorem in a cone, which is a classical tool extensively used in the related literature (see, for instance, [1, 3-5] and references therein). We will use cones of the form

$$
\mathcal{K}=\{x \in \mathcal{C}([0, T],[0, \infty)): \varphi(x) \geq \sigma\|x\|\},
$$

where $0<\sigma \leq 1$ is a fixed constant and $\varphi: \mathcal{C}([0, T],[0, \infty)) \rightarrow[0, \infty)$ is a functional satisfying

(i) $\varphi(x+y) \geq \varphi(x)+\varphi(y)$ for all $x, y \in \mathcal{C}([0, T],[0, \infty))$,

(ii) $\varphi(\lambda x)=\lambda \varphi(x)$ for all $\lambda>0$ and $x \in \mathcal{C}([0, T],[0, \infty))$.

In particular, in Section 2, we use the standard choice $\varphi(x)=\min _{t \in[0, T]} x(t)$, and in Section 3, we use $\varphi(x)=\int_{0}^{T} x(s) d s$, which has been recently introduced in [3].

We say that the linear problem

$$
x^{\prime \prime}+a(t) x=0, \quad x(0)=x(T), \quad x^{\prime}(0)=x^{\prime}(T)
$$

is nonresonant when its unique solution is the trivial one. It is well known that if (1.5) is nonresonant, then the nonhomogeneous problem

$$
x^{\prime \prime}+a(t) x=h(t), \quad \text { a.e. } t \in[0, T] ; \quad x(0)=x(T), \quad x^{\prime}(0)=x^{\prime}(T),
$$

always has a unique solution which, moreover, can be written as

$$
x(t)=\int_{0}^{T} G(t, s) h(s) d s,
$$

where $G(t, s)$ is Green's function related to (1.5). Thus, defining for each $\lambda>0$ the operator

$$
\tau_{\curlywedge}: \Phi\left(\tau_{\curlywedge}\right) \equiv\{x \in \mathcal{C}([0, T]): x(t)>0 \forall t \in[0, T]\} \longrightarrow \mathcal{C}([0, T])
$$

given by

$$
\tau_{\curlywedge} x(t)=\lambda \int_{0}^{T} G(t, s) g(s) f(x(s)) d s+\int_{0}^{T} G(t, s) c(s) d s, \quad t \in[0, T],
$$

we have that $x>0$ is a solution of problem (1.2) if and only if $x=\tau_{\lambda} x$. 
Throughout the paper, we will use the following notation:

$$
\begin{gathered}
r(t)=\int_{0}^{T} G(t, s) c(s) d s, \\
m=\min _{t, s \in[0, T]} G(t, s), \quad M=\max _{t, s \in[0, T]} G(t, s), \\
f_{0}=\lim _{x \rightarrow 0^{+}} \frac{f(x)}{x}, \quad f_{\infty}=\lim _{x \rightarrow \infty} \frac{f(x)}{x} .
\end{gathered}
$$

For $a \in L^{1}(0, T)$, we denote by $a_{+}=\max \{a, 0\}$ its positive part, and for $1 \leq p \leq \infty$, we denote by $\hat{p}$ its conjugate, that is $1 / p+1 / \hat{p}=1$. Moreover, for an essentially bounded function $h:[0, T] \rightarrow \mathbb{R}$, we define

$$
h_{*}=\inf \underset{t \in[0, T]}{\operatorname{ess}} h(t), \quad h^{*}=\sup \underset{t \in[0, T]}{\operatorname{ess}} h(t),
$$

and for $x \in \mathcal{C}([0, T])$, we will define

$$
\|x\|=\sup _{t \in[0, T]}|x(t)|
$$

The following section is devoted to prove the existence, multiplicity, and nonexistence of solutions of problem (1.2) by assuming that the related Green's function is strictly positive, whereas in Section 3, we turn out to the case, where the related Green's function is non negative. We point out that in the recent paper [6], the existence of solution for problem (1.2) with a sign-changing Green's function is studied, but only considering a regular $f$ and $c(t) \equiv 0$.

\section{Positive Green's Function}

In this section, we assume the following hypotheses:

(H0) $\gamma_{*}>0$ or $c(t) \equiv 0$,

(H1) problem (1.5) is nonresonant and the corresponding Green's function $G(t, s)$ is strictly positive on $[0, T] \times[0, T]$,

(H2) $g \in L^{1}([0, T]), g(t) \geq 0$ for a.e. $t \in[0, T]$, and $\int_{0}^{T} g(s) d s>0$,

(H3) $f:(0, \infty) \rightarrow(0, \infty)$ is continuous,

(H4) $c \in L^{1}([0, T])$.

Notice that condition (H3) allows $f$ to be singular at $x=0$. In particular, (H3) is satisfied when $f(x)=1 / x^{\alpha}, \alpha>0$ (the case $0<\alpha<1$ is called a weak singularity, while $\alpha \geq 1$ is called an strong singularity).

On the other hand, it is well known that for constant $a(t) \equiv k^{2}$, condition (H1) is equivalent to $0<k<\pi / T$. For a time-dependent and nonnegative potential $a(t)$, Torres gave a sharp $L^{p}$-criterium [5] based on an antimaximum principle obtained in a previous work by Torres and Zhang [7]. That criterium has been extended in [8] for sign-changing potentials with strictly positive average. The obtained result is the following. 
Proposition 2.1 (see [8, Theorem 3.2]). Define

$$
K(\alpha, T)= \begin{cases}\frac{2 \pi}{\alpha T^{1+2 / \alpha}}\left(\frac{2}{2+\alpha}\right)^{1-2 / \alpha}\left(\frac{\Gamma(1 / \alpha)}{\Gamma(1 / 2+1 / \alpha)}\right)^{2}, & \text { if } 1 \leq \alpha<\infty, \\ \frac{4}{T}, & \text { if } \alpha=\infty,\end{cases}
$$

where $\Gamma$ is the usual Gamma function.

Assume that $a \in L^{p}(0, T)$ for some $1 \leq p \leq \infty, \int_{0}^{T} a(t) d t>0$, and moreover,

$$
\left\|a_{+}\right\|_{p}<K(2 \widehat{p}, T) \text {. }
$$

Then, $G(t, s)>0$ for all $(t, s) \in[0, T] \times[0, T]$.

In [9], by studying antimaximum principles for the semilinear equation

$$
\left(\left|u^{\prime}\right|^{p-2} u^{\prime}\right)^{\prime}+a(t)\left(|u|^{p-2} u\right)=h(t), \quad u(0)=u(T), \quad u^{\prime}(0)=u^{\prime}(T),
$$

the previous result has been extended to the potentials with nonnegative average as follows.

Lemma 2.2 (see [9, Theorem 3.4 and Remark 3.7]). Assume that $a \in L^{p}(I)$ for some $p \geq 1$, $\int_{0}^{T} a(t) d t \geq 0$, and moreover,

$$
\left\|a_{+}\right\|_{p} \leq K(2 \hat{p}, T) .
$$

Then, $G(t, s)>0$ for a.e. $(t, s) \in I \times I$.

Zhang constructs in [10] some examples of potentials a $(t)$ for which the related Green's function is strictly positive, but (2.2) does not hold. In consequence, the best Sobolev constant $K(2 \hat{p}, T)$ is not an optimal estimate to ensure the positiveness of Green's function. For optimal conditions in order to get maximum or antimaximum principles, expressed using eigenvalues, Green's functions, or rotation numbers, the reader is referred to the recent work of Zhang [11].

Example 2.3. By Proposition 2.1, Hill's equation

$$
x^{\prime \prime}+a(1+b \cos (t)) x=0,
$$

with the periodic boundary conditions

$$
x(0)=x(2 \pi), \quad x^{\prime}(0)=x^{\prime}(2 \pi)
$$

satisfies (H1), provided that $a>0$, and moreover,

$$
\left\|a(1+b \cos (t))_{+}\right\|_{p}<K(2 \widehat{p}, 2 \pi), \quad \text { for some } p \in[1, \infty],
$$




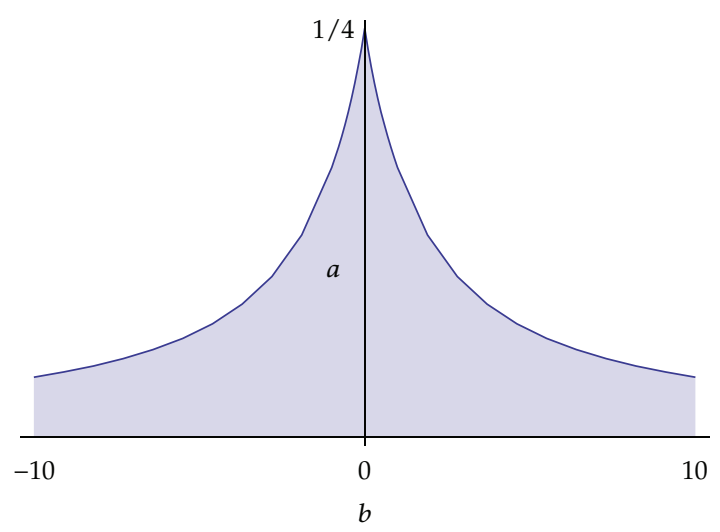

Figure 1: Graphic of $M(b)$.

where $K$ is given by (2.1). So, for each $b \in \mathbb{R}$, the condition (H1) is fulfilled if

$$
0<a<M(b):=\sup _{p \in[1, \infty]}\left\{\frac{K(2 \hat{p}, 2 \pi)}{\left\|(1+b \cos (t))_{+}\right\|_{p}}\right\}
$$

In particular, it is known that $M(0)=1 / 4$ and $M(1)=0.16448$ (since the maximum of $K(2 \widehat{p}, 2 \pi) /\|1+\cos (t)\|_{p}$ is attained at $p \approx 2.1941$, see [10, Example 4.4]). The graphic of $M(b)$ is showed in Figure 1.

From (H1), it follows that $m>0$, and we define the cone

$$
\mathcal{K}:=\left\{x \in \mathcal{C}([0, T],[0, \infty)): \min _{t \in[0, T]} x(t) \geq \sigma\|x\|\right\}
$$

where

$$
\sigma=\min \left\{\frac{m}{M}, \frac{\gamma_{*}}{\gamma^{*}}\right\}, \quad \text { if } \gamma_{*}>0 \quad \text { or } \quad \sigma=\frac{m}{M}, \quad \text { if } c(t) \equiv 0
$$

In both cases, $0<\sigma<1$, and for $0<r<R$, we define

$$
\mathcal{K}_{r, R}:=\{x \in \mathcal{K}: r \leq\|x\| \leq R\}
$$

Next, we give sufficient conditions for the solvability of problem (1.2).

Theorem 2.4. Assume that conditions (H0), (H1), (H2), (H3), and (H4) are fulfilled. Then, for each $\lambda>0$ and $0<r<R$, the operator $\tau_{\lambda}: \mathcal{K}_{r, R} \rightarrow \mathcal{K}$ given by (1.9) is well defined and completely continuous. 
Moreover, if either

(i) $\left\|\tau_{\curlywedge} x\right\| \leq\|x\|$ for any $x \in \mathcal{K}$ with $\|x\|=r$ and $\left\|\tau_{\curlywedge} x\right\| \geq\|x\|$ for any $x \in \mathcal{K}$ with $\|x\|=R$, or

(ii) $\left\|\tau_{\lambda} x\right\| \geq\|x\|$ for any $x \in \mathcal{K}$ with $\|x\|=r$ and $\left\|\tau_{\lambda} x\right\| \leq\|x\|$ for any $x \in \mathcal{K}$ with $\|x\|=R$, then $\tau_{\lambda}$ has a fixed point in $\mathcal{K}_{r, R}$, which is a positive solution of problem (1.2).

Proof. Note that if $x \in \mathcal{K}_{r, R}$, then $0<\sigma r \leq x(t) \leq R$ for all $t \in[0, T]$, so $\mathcal{K}_{r, R} \subset \boldsymbol{\Phi}\left(\mathcal{\tau}_{\lambda}\right)$, and then $\tau_{\lambda}: \mathcal{K}_{r, R} \rightarrow \mathcal{C}([0, T])$ is well defined. Standard arguments show that $\mathcal{\tau}_{\lambda}\left(\boldsymbol{\Phi}\left(\boldsymbol{\tau}_{\lambda}\right)\right) \subset \mathcal{K}$ and that $\tau_{\lambda}$ is completely continuous. Then, from Krasnoselskii's fixed point theorem (see $\left[12\right.$, p. 148]), it follows the existence of a fixed point for $\tau_{\lambda}$ in $\mathcal{K}_{r, R}$ which is, by the definition of $\tau_{\lambda}$, a positive solution of problem (1.2).

Before proving the existence and multiplicity results for problem (1.2), we need some technical lemmas proved in the next subsection.

\subsection{Auxiliary Results}

Lemma 2.5. Assume that conditions (H0), (H1), (H2), (H3), and (H4) are satisfied. Then, for each $R>\gamma^{*}$, there exists $\lambda_{0}(R)>0$ such that for every $0<\lambda \leq \lambda_{0}(R)$, we have

$$
\left\|\tau_{\lambda} x\right\| \leq\|x\| \quad \text { for } x \in \mathcal{K} \text { with }\|x\|=R \text {. }
$$

Proof. Fix $R>\gamma^{*}$, and let $x \in \mathcal{K}$ with $\|x\|=R$. If

$$
0<\lambda \leq \lambda_{0}(R):=\frac{R-\gamma^{*}}{M \max _{u \in[\sigma R, R]} f(u) \int_{0}^{T} g(s) d s}
$$

then, for all $t \in[0, T]$ the following inequalities hold:

$$
\begin{aligned}
\tau_{\lambda} x(t) & =\lambda \int_{0}^{T} G(t, s) g(s) f(x(s)) d s+\gamma(t) \\
& \leq \lambda M \max _{u \in[\sigma R, R]} f(u) \int_{0}^{T} g(s) d s+\gamma^{*} \\
& \leq R=\|x\|,
\end{aligned}
$$

and thus $\left\|\tau_{\lambda} x\right\| \leq\|x\|$.

Lemma 2.6. Assume that conditions (H0), (H1), (H2), (H3), and (H4) are fullfiled. Then, for each $r>0$, there exists $\lambda_{0}(r)>0$ such that for every $\lambda \geq \lambda_{0}(r)$, we have

$$
\left\|\tau_{\lambda} x\right\| \geq\|x\|, \quad \text { for } x \in \mathcal{K} \text { with }\|x\|=r \text {. }
$$


Proof. Fix $r>0$, and let $x \in \mathcal{K}$ with $\|x\|=r$. If

$$
\lambda \geq \lambda_{0}(r):=\frac{r}{m \min _{u \in[\sigma r, r]} f(u) \int_{0}^{T} g(s) d s},
$$

then

$$
\begin{aligned}
\tau_{\curlywedge} x(t) & =\lambda \int_{0}^{T} G(t, s) g(s) f(x(s)) d s+\gamma(t) \\
& \geq \lambda m \min _{u \in[\sigma R, R]} f(u) \int_{0}^{T} g(s) d s+\gamma_{*} \\
& \geq r=\|x\|,
\end{aligned}
$$

and thus $\left\|\tau_{\curlywedge} x\right\| \geq\|x\|$.

Lemma 2.7. Suppose that conditions $(H 1),(H 2),(H 3)$, and $(H 4)$ are satisfied and $c(t) \equiv 0$. Then, if $f_{0}=0$, there exists $r_{0}(\lambda)>0$ such that for every $0<r \leq r_{0}(\lambda)$, we have

$$
\left\|\tau_{\lambda} x\right\| \leq\|x\|, \quad \text { for } x \in \mathcal{K} \text { with }\|x\|=r .
$$

Proof. Since $f_{0}=0$ for $\varepsilon=\varepsilon(\lambda)=1 / \lambda M \int_{0}^{T} g(s) d s$, there exists $r_{0}(\lambda)>0$ such that $f(u) \leq \varepsilon u$ for each $0<u \leq r_{0}(\lambda)$.

Fix $0<r \leq r_{0}(\lambda)$, and let $x \in \mathcal{K}$ with $\|x\|=r$. Then,

$$
\begin{aligned}
\tau_{\curlywedge} x(t) & =\lambda \int_{0}^{T} G(t, s) g(s) f(x(s)) d s \\
& \leq \lambda M \int_{0}^{T} g(s) \varepsilon x(s) d s \\
& \leq \lambda M \varepsilon\|x\| \int_{0}^{T} g(s) d s \\
& =\|x\|,
\end{aligned}
$$

and thus $\left\|\tau_{\lambda} x\right\| \leq\|x\|$.

Lemma 2.8. Assume that hypothesis $(H 0),(H 1),(H 2),(H 3)$, and $(H 4)$ hold. Then, if $f_{0}=\infty$, there exists $r_{0}(\lambda)>0$ such that for every $0<r \leq r_{0}(\lambda)$, we have

$$
\left\|\tau_{\curlywedge} x\right\| \geq\|x\|, \quad \text { for } x \in \mathcal{K} \text { with }\|x\|=r .
$$

Proof. Since $f_{0}=\infty$ for $L=L(\lambda)=1 / \lambda m \sigma \int_{0}^{T} g(s) d s$, there exists $r_{0}(\lambda)>0$ such that $f(u) \geq L u$ for each $0<u \leq r_{0}(\lambda)$. 
Fix $0<r \leq r_{0}(\lambda)$, and let $x \in \mathcal{K}$ with $\|x\|=r$. Then,

$$
\begin{aligned}
\tau_{\curlywedge} x(t) & =\lambda \int_{0}^{T} G(t, s) g(s) f(x(s)) d s+\gamma(t) \\
& \geq \lambda m \int_{0}^{T} g(s) L x(s) d s+\gamma_{*} \\
& \geq \lambda m L \sigma\|x\| \int_{0}^{T} g(s) d s \\
& =\|x\|,
\end{aligned}
$$

and thus $\left\|\tau_{\lambda} x\right\| \geq\|x\|$.

Lemma 2.9. Suppose that conditions $(H 0),(H 1),(H 2),(H 3)$, and (H4) are satisfied. Then, if $f_{\infty}=0$ then, there exists $R_{0}(\lambda)>0$ such that for every $R \geq R_{0}(\lambda)$, we have

$$
\left\|\tau_{\curlywedge} x\right\| \leq\|x\|, \text { for } x \in \mathcal{K} \text { with }\|x\|=R \text {. }
$$

Proof. Since $f_{\infty}=0$ for $\varepsilon(\lambda)=1 / 2 \lambda M \int_{0}^{T} g(s) d s$, there exists $R_{1}(\lambda)>0$ such that $f(u) \leq \varepsilon u$ for each $u \geq R_{1}(\lambda)$. We define $R_{0}(\lambda):=\max \left\{R_{1}(\lambda) / \sigma, 2 \gamma^{*}\right\}$.

Fix $R \geq R_{0}(\lambda)$, and let $x \in \mathcal{K}$ with $\|x\|=R$. Then,

$$
\begin{aligned}
\tau_{\lambda} x(t) & =\lambda \int_{0}^{T} G(t, s) g(s) f(x(s)) d s+\gamma(t) \\
& \leq \lambda M \int_{0}^{T} g(s) \varepsilon x(s)+\gamma^{*} \\
& \leq \lambda M \varepsilon\|x\| \int_{0}^{T} g(s) d s+\gamma^{*} \\
& =\frac{R}{2}+\gamma^{*} \leq \frac{R}{2}+\frac{R}{2}=R=\|x\|,
\end{aligned}
$$

and thus $\left\|\tau_{\curlywedge} x\right\| \leq\|x\|$.

Lemma 2.10. Assume that $(H 0),(H 1),(H 2),(H 3)$, and $(H 4)$ are fullfiled. Then, if $f_{\infty}=\infty$, there exists $R_{0}(\lambda)>0$ such that for every $R \geq R_{0}(\lambda)$, we have

$$
\left\|\tau_{\lambda} x\right\| \geq\|x\|, \quad \text { for } x \in \mathcal{K} \text { with }\|x\|=R \text {. }
$$

Proof. Since $f_{\infty}=\infty$ for $L=L(\lambda)=1 / \lambda m \sigma \int_{0}^{T} g(s) d s$, there exists $R_{1}(\lambda)>0$ such that $f(u) \geq$ $L u$ for each $u \geq R_{1}(\lambda)$. We define $R_{0}(\lambda):=R_{1}(\lambda) / \sigma$. 
Fix $R \geq R_{0}(\lambda)$, and let $x \in \mathcal{K}$ with $\|x\|=R$. Then,

$$
\begin{aligned}
\tau_{\lambda} x(t) & =\lambda \int_{0}^{T} G(t, s) g(s) f(x(s)) d s+\gamma(t) \\
& \geq \lambda m \int_{0}^{T} g(s) L x(s) d s+\gamma_{*} \\
& \geq \lambda m L \sigma\|x\| \int_{0}^{T} g(s) d s \\
& =\|x\|,
\end{aligned}
$$

and thus $\left\|\tau_{\lambda} x\right\| \geq\|x\|$.

In the sequel, we study separately the two different cases considered in condition $(H 0)$; that is, $\gamma_{*}>0$ or $c(t) \equiv 0$.

\subsection{The Case $\gamma_{*}>0$}

Theorem 2.11. Assume that conditions (H1), (H2), (H3), and (H4) are fulfilled. If, moreover, $\gamma_{*}>0$, the following results hold:

(1) there exists $\lambda_{0}>0$ such that problem (1.2) has a positive solution if $0<\lambda<\lambda_{0}$,

(2) if $f_{\infty}=0$, then problem (1.2) has a positive solution for every $\lambda>0$,

(3) if $f_{\infty}=\infty$, then there exists $\lambda_{0}>0$ such that problem (1.2) has two positive solutions if $0<\lambda<\lambda_{0}$

(4) if $f_{0}>0$ and $f_{\infty}>0$, then there exists $\lambda_{0}>0$ such that problem (1.2) has no positive solutions if $\lambda>\lambda_{0}$.

Proof. Fix $0<r<\gamma_{*}$. Then, for each $\lambda>0$ and $x \in \mathcal{K}$ with $\|x\|=r$, we have

$$
\begin{aligned}
\left\|\tau_{\lambda} x\right\| \geq \tau_{\lambda} x(t) & =\lambda \int_{0}^{T} G(t, s) g(s) f(x(s)) d s+\gamma(t) \\
& \geq \gamma_{*}>r=\|x\| .
\end{aligned}
$$

Part 1. Fix $R>\gamma^{*}\left(\geq \gamma_{*}>r\right)$, and take $\lambda_{0}=\lambda_{0}(R)$ given by Lemma 2.5. Then, from Theorem 2.4 (ii), it follows the existence of a positive solution for problem (1.2) if $0<\lambda<\lambda_{0}$.

Part 2. Fix $\lambda>0$, and take $R>\max \left\{r, R_{0}(\lambda)\right\}$, where $R_{0}(\lambda)$ is given by Lemma 2.9. Then, from Theorem 2.4 (ii), it follows the existence of a positive solution for problem (1.2).

Part 3. Fix $R_{2}>R_{1}>\gamma^{*}\left(\geq \gamma_{*}>r\right)$, and take $\lambda_{0}=\min \left\{\lambda_{0}\left(R_{1}\right), \lambda_{0}\left(R_{2}\right)\right\}$, where $\lambda_{0}\left(R_{1}\right)$ and $\lambda_{0}\left(R_{2}\right)$ are the given by Lemma 2.5 . 
Now, fix $0<\lambda<\lambda_{0}$, and take $R>\max \left\{R_{2}, R_{0}(\lambda)\right\}$, where $R_{0}(\lambda)$ is given by Lemma 2.10. Therefore, from Theorem 2.4, it follows the existence of two positive solutions $x_{1}$ and $x_{2}$ for problem (1.2) such that

$$
r \leq\left\|x_{1}\right\| \leq R_{1}<R_{2} \leq\left\|x_{2}\right\| \leq R .
$$

Part 4. Since $f_{0}>0$ and $f_{\infty}>0$, there exists $L>0$ such that $f(u) \geq L u$ for all $u>0$. Define

$$
\lambda_{0}:=\frac{1}{m \sigma L \int_{0}^{T} g(s) d s}
$$

If for $\lambda>\lambda_{0}$, there exists a positive solution $x$ of problem (1.2), we know that $x \in \Phi\left(\tau_{\lambda}\right)$ and, as consequence, $x=\tau_{\lambda}(x) \in \mathcal{K}$. Therefore, we deduce the following inequalities:

$$
\begin{aligned}
\|x\| & =\left\|\tau_{\lambda} x\right\| \geq \tau_{\lambda} x(t)=\lambda \int_{0}^{T} G(t, s) g(s) f(x(s)) d s+\gamma(t) \\
& \geq \lambda m \int_{0}^{T} g(s) L x(s) d s+\gamma_{*} \\
& \geq \lambda m L \sigma\|x\| \int_{0}^{T} g(s) d s \\
& >\|x\|,
\end{aligned}
$$

and we attain a contradiction.

Example 2.12. Let us consider the forced Mathieu-Duffing-type equation

$$
x^{\prime \prime}+a(1+b \cos (t)) x-\lambda x^{3}=c(t)
$$

which fits into expression (1.2) by defining $a(t)=a(1+b \cos (t)), g(t)=1$ and $f(x)=x^{3}$.

Equation (2.30), with $c(t) \equiv 0$, was studied in [13], where a sufficient condition for the existence of a $2 \pi$-periodic solution is given. However, since the proof relies in the application of Schauder's fixed point theorem in a ball centered at the origin, the trivial solution $x(t) \equiv 0$ is not excluded. The existence of a nontrivial solution was later obtained by Torres in [5, Corollary 4.2]. More precisely, Torres proves that if function $a(t)>0$ for a.e. $t \in[0,2 \pi]$ and $\|a\|_{p}<K(2 \hat{p}, 2 \pi)$, then the homogeneous problem $(c(t) \equiv 0)(2.30)$ has at least two nontrivial one-signed $2 \pi$-periodic solutions.

In this paper, as a consequence of Example 2.3 and Theorem 2.11, Part 3, we arrive at the following multiplicity result for the inhomogeneous $(c(t) \neq 0)$ equation $(2.30)$ with a not necessarily constant sign function $a(t)$.

Corollary 2.13. If condition (2.8) is satisfied and $\gamma_{*}>0$, then there exists $\lambda_{0}>0$ such that (2.30) has at least two positive $2 \pi$-periodic solutions, provided that $0<\lambda<\lambda_{0}$. 


\subsection{The Case $c(t) \equiv 0$}

Theorem 2.14. Assume that conditions $(H 1),(H 2),(H 3)$, and $(H 4)$ hold. If moreover $c(t) \equiv 0$ the following results hold:

(1) if $f_{0}=\infty$ or $f_{\infty}=\infty$, then there exists $\lambda_{0}>0$ such that problem (1.2) has a positive solution if $0<\lambda<\lambda_{0}$,

(2) if $f_{\infty}=0$ then there exists $\lambda_{0}>0$ such that problem (1.2) has a positive solution for every $\lambda>\lambda_{0}$

(3) if $f_{0}=\infty$ and $f_{\infty}=0$ then problem (1.2) has a positive solution for every $\lambda>0$,

(4) if $f_{0}=\infty$ and $f_{\infty}=\infty$ then there exists $\lambda_{0}>0$ such that problem (1.2) has two positive solutions if $0<\lambda<\lambda_{0}$,

(5) if $f_{0}=0$ and $f_{\infty}=\infty$, then problem (1.2) has a positive solution for every $\lambda>0$,

(6) if $f_{0}=0$ and $f_{\infty}=0$ then there exists $\lambda_{0}>0$ such that problem (1.2) has two positive solutions if $\lambda>\lambda_{0}$,

(7) if $f_{0}>0$ and $f_{\infty}>0$, then there exists $\lambda_{0}>0$ such that problem (1.2) has no positive solutions if $\lambda>\lambda_{0}$.

Proof of Part 1. Fix $R>\gamma^{*}=0$ and take $\lambda_{0}=\lambda_{0}(R)>0$ given by Lemma 2.5. In consequence, for all $0<\lambda \leq \lambda_{0}(R)$ we have

$$
\left\|\tau_{\curlywedge} x\right\| \leq\|x\|, \quad \text { for } x \in \mathcal{K} \text { with }\|x\|=R
$$

Now, let $0<\lambda<\lambda_{0}$ be fixed, and choose $0<r<\min \left\{R, r_{0}(\lambda)\right\}$, where $r_{0}(\lambda)$ is given by Lemma 2.8 when $f_{0}=\infty$. In case of $f_{\infty}=\infty$, we get $r>\max \left\{R, R_{0}(\lambda)\right\}>R$, with $R_{0}(\lambda)$ given by Lemma 2.10. In both situations, we arrive at

$$
\left\|\tau_{\lambda} x\right\| \geq\|x\|, \quad \text { for } x \in \mathcal{K} \text { with }\|x\|=r
$$

Thus, Theorem 2.4 implies the existence of a positive solution for problem (1.2).

Part 2. Fix $r>0$, and take $\lambda_{0}=\lambda_{0}(r)>0$ given by Lemma 2.6. Now, for each $\lambda>\lambda_{0}$, take $R>\max \left\{r, R_{0}(\lambda)\right\}$, with $R_{0}(\lambda)$ given by Lemma 2.9 , and apply Theorem 2.4 .

Part 3. For each $\lambda>0$, take $r_{0}(\lambda)<R_{0}(\lambda)$ given by Lemmas 2.8 and 2.9, respectively, and apply Theorem 2.4 .

Part 4. Fix $R_{2}>R_{1}>\gamma^{*}=0$, and take $\lambda_{0}=\min \left\{\lambda_{0}\left(R_{1}\right), \lambda_{0}\left(R_{2}\right)\right\}$ given by Lemma 2.5. Now, for each $0<\lambda<\lambda_{0}$, take $r<\min \left\{R_{1}, r_{0}(\lambda)\right\}$ given by Lemma 2.8 and $R>$ $\max \left\{R_{2}, R_{0}(\lambda)\right\}$ given by Lemma 2.10. Then, Theorem 2.4 implies the existence of two positive solutions $x_{1}$ and $x_{2}$ for problem (1.2) such that

$$
r \leq\left\|x_{1}\right\| \leq R_{1}<R_{2} \leq\left\|x_{2}\right\| \leq R
$$

Part 5. Use Lemmas 2.7 and 2.10 and Theorem 2.4. 
Part 6. Use Lemmas 2.6, 2.7, and 2.9 and Theorem 2.4 twice.

Part 7. The proof follows the same steps as Part 4 in Theorem 2.11.

Remark 2.15. Theorem 2.14 complements [1, Theorem 2.1], since it provides similar results for the problem $x^{\prime \prime}+k^{2} x=\lambda g(t) f(x)$, with $0<k<\pi / T$.

Example 2.16. Consider as a model the problem

$$
x^{\prime \prime}+a(1+b \cos (t)) x=\lambda\left(\frac{1}{x^{\alpha}}+\mu x^{\beta}\right), \quad x(0)=x(2 \pi), \quad x^{\prime}(0)=x^{\prime}(2 \pi),
$$

where $a>0, b \in \mathbb{R}$ and $\alpha, \beta, \mu \geq 0$. When $b=1$ and $\mu=0,(2.34)$ is the Brillouinbeam focusing equation which has been widely studied in the literature (see $[5,10,14]$ and references therein). Now, we have the following:

Corollary 2.17. Assume condition (2.8). Then, the following results are satisfied:

(i) if $0 \leq \beta<1$, then problem (2.34) has a positive solution for every $\lambda>0$.

(ii) if $\beta=1$ and $\mu>0$, then there exists $\lambda_{0}>0$ such that problem (2.34) has a positive solution for every $0<\lambda<\lambda_{0}$, and there exists $\lambda_{1}>0$ such that the problem has no positive solution for $\lambda>\lambda_{1}$.

(iii) if $\beta>1$ and $\mu>0$, then there exists $\lambda_{0}>0$ such that problem (2.34) has two positive solutions for every $0<\lambda<\lambda_{0}$.

Proof. Condition (2.8) implies that condition (H1) is satisfied. Now, to prove (i), (ii) or (iii) it is enough to apply Theorem 2.14 Part 3, Part 1 and Part 7 or Part 4, respectively.

\section{Nonnegative Green's Function}

In this section instead of conditions $(H 1)$ and $(H 3)$, we assume

$\widetilde{(H 1)}$ Problem (1.5) is nonresonant, the corresponding Green's function $G(t, s)$ is nonnegative on $[0, T] \times[0, T]$, and $\beta=\min _{t \in[0, T]} \int_{0}^{T} G(t, s) d s>0$,

$\widetilde{(H 3)} f:[0, \infty) \rightarrow[0, \infty)$ is continuous, and $f(u)>0$ for all $u>0$.

Notice that $x(t)=\int_{0}^{T} G(t, s) d s$ is the unique solution of the problem

$$
x^{\prime \prime}(t)+a(t) x(t)=1, \quad x(0)=x(T), \quad x^{\prime}(0)=x^{\prime}(T),
$$

and then $\widetilde{(H 1)}$ asks for this solution to be strictly positive. On the other hand, assumption $\widetilde{(H 3)}$ allows us to consider only regular problems. We will discuss to singular problems in Section 3.1 by means of a truncation technique.

For constant $a(t) \equiv k^{2}$, condition $\widetilde{(H 1)}$ is equivalent to $0<k \leq \pi / T$. For nonconstant $a(t)$, condition $\widetilde{(H 1)}$ is satisfied provided that Lemma 2.2 holds.

On the other hand, under condition $\widetilde{(H 1)}$, it is allowed that

$$
m=\min _{t, s \in[0, T]} G(t, s)=0,
$$


so $\sigma=m / M$ can be equal to 0 , and thus, the arguments used in the previous section do not work. So, by assuming that $\gamma_{*} \geq 0$, let us define

$$
\widetilde{\mathcal{K}}:=\left\{x \in \mathcal{C}([0, T],[0, \infty)): \int_{0}^{T} x(s) d s \geq \tilde{\sigma}\|x\|\right\},
$$

where $\tilde{\sigma}=\min \left\{\beta / T M, \int_{0}^{T} \gamma(s) d s / T\|\gamma\|\right\}$ if $\|\gamma\|>0$ or $\widetilde{\sigma}=\beta / T M$ if $\|\gamma\|=0$. As far as we know, the cone $\widetilde{\mathcal{K}}$ was introduced in [3].

Clearly, $0<\tilde{\sigma} \leq 1$, and for $0<r<R$, we define

$$
\widetilde{\mathcal{K}}_{r, R}:=\{x \in \widetilde{\mathcal{K}}: r \leq\|x\| \leq R\} .
$$

Next, we prove the following result similar to Theorem 2.4.

Theorem 3.1. Assume that $(H 0), \widetilde{(H 1)},(H 2), \widetilde{(H 3)}$, and $(H 4)$ hold. Then, for each $\lambda>0$ and $0<r<R$, the operator $\tau_{\lambda}: \widetilde{\mathcal{K}}_{r, R} \rightarrow \widetilde{\mathcal{K}}$ given by (1.9) is well defined and completely continuous.

Moreover, if either

(i) $\left\|\tau_{\lambda} x\right\| \leq\|x\|$ for any $x \in \widetilde{\mathcal{K}}$ with $\|x\|=r$ and $\left\|\tau_{\lambda} x\right\| \geq\|x\|$ for any $x \in \widetilde{\mathcal{K}}$ with $\|x\|=R$, or

(ii) $\left\|\tau_{\lambda} x\right\| \geq\|x\|$ for any $x \in \widetilde{\mathcal{K}}$ with $\|x\|=r$ and $\left\|\tau_{\lambda} x\right\| \leq\|x\|$ for any $x \in \widetilde{\mathcal{K}}$ with $\|x\|=R$, then $\tau_{\lambda}$ has a fixed point in $\widetilde{\mathcal{K}}_{r, R}$, which is a nonnegative solution of problem (1.2).

Proof. If $x \in \mathcal{C}([0, T],[0, \infty))$ and assuming $\|\gamma\|>0$ (the case $\|\gamma\|=0$ being analogous), we obtain

$$
\begin{aligned}
\int_{0}^{T} \tau_{\curlywedge} x(t) d t & =\lambda \iint_{0}^{T} G(t, s) g(s) f(x(s)) d s d t+\int_{0}^{T} \gamma(t) d t \\
& =\lambda \int_{0}^{T} g(s) f(x(s))\left(\int_{0}^{T} G(t, s) d t\right) d s+\int_{0}^{T} \gamma(s) d s \\
& \geq \lambda \beta \int_{0}^{T} g(s) f(x(s)) d s+\int_{0}^{T} \gamma(s) d s \\
& =\lambda \frac{\beta}{T M} T M \int_{0}^{T} g(s) f(x(s)) d s+\frac{\int_{0}^{T} \gamma(s) d s}{T\|\gamma\|} T\|r\| \\
& \geq \tilde{\sigma}\left(\lambda T M \int_{0}^{T} g(s) f(x(s)) d s+T\|\gamma\|\right) \\
& \geq \tilde{\sigma}\left\|\tau_{\lambda} x\right\| .
\end{aligned}
$$

Thus, $\tau_{\lambda}(\mathcal{C}([0, T],[0, \infty))) \subset \widetilde{\mathcal{K}}$, and it is standard to show that $\tau_{\lambda}$ is completely continuous. In consequence, from Krasnoselskii's fixed point theorem (see [12, p.148]), it follows the 
existence of a fixed point for $\tau_{\lambda}$ in $\widetilde{\mathcal{K}}_{r, R}$ which it is, by the definition of $\tau_{\lambda}$, a non negative solution of problem (1.2).

Now, we are going to give sufficient conditions to obtain $\left\|\tau_{\lambda} x\right\| \leq\|x\|$ or $\left\|\tau_{\lambda} x\right\| \geq\|x\|$. The combination of the next lemmas with Theorem 3.1 will allow us to prove existence and multiplicity results for problem (1.2).

Lemma 3.2. Suppose that the conditions $(H 0), \widetilde{(H 1)},(H 2), \widetilde{(H 3)}$, and $(H 4)$ are satisfied. Then, for each $R>\gamma^{*}$, there exists $\lambda_{0}(R)>0$ such that for every $0<\lambda \leq \lambda_{0}(R)$, we have

$$
\left\|\tau_{\lambda} x\right\| \leq\|x\|, \quad \text { for } x \in \widetilde{\mathcal{K}} \text { with }\|x\|=R \text {. }
$$

Proof. Fix $R>r^{*}$, and let $x \in \widetilde{\mathcal{K}}$ with $\|x\|=R$. If

$$
0<\lambda \leq \lambda_{0}(R):=\frac{R-\gamma^{*}}{M \max _{u \in[0, R]} f(u) \int_{0}^{T} g(s) d s},
$$

then

$$
\begin{aligned}
\tau_{\curlywedge} x(t) & =\lambda \int_{0}^{T} G(t, s) g(s) f(x(s)) d s+\gamma(t) \\
& \leq \lambda M \max _{u \in[0, R]} f(u) \int_{0}^{T} g(s) d s+\gamma^{*} \\
& \leq R=\|x\|,
\end{aligned}
$$

and thus $\left\|\tau_{\curlywedge} x\right\| \leq\|x\|$.

Lemma 3.3. Assume that $(\widetilde{H 1}),(H 2),(\widetilde{H 3}),(H 4)$, and $\int_{0}^{T} \gamma(s) d s>0$ are satisfied. Then, there exists $r_{0}>0$ such that for each $0<r<r_{0}$, we have

$$
\left\|\tau_{\lambda} x\right\| \geq\|x\|, \quad \text { for } x \in \widetilde{\mathcal{K}} \text { with }\|x\|=r .
$$

Proof. Fix $0<r<r_{0}:=1 / T \int_{0}^{T} \gamma(s) d s$, and let $x \in \widetilde{\mathcal{K}}$ with $\|x\|=r$. Then,

$$
\begin{aligned}
\left\|\tau_{\lambda} x\right\| \geq \frac{1}{T} \int_{0}^{T} \tau_{\lambda} x(t) d t & =\frac{\lambda}{T} \iint_{0}^{T} G(t, s) g(s) f(x(s)) d s d t+\frac{1}{T} \int_{0}^{T} \gamma(t) d t \\
& \geq r_{0}>r=\|x\|,
\end{aligned}
$$

and thus $\left\|\tau_{\curlywedge} x\right\| \geq\|x\|$. 
Lemma 3.4. Let $(H 0),(\widetilde{H 1}),(H 2),(\widetilde{H 3})$, and $(H 4)$ be fulfilled. Then, if $f_{\infty}=0$, there exists $R_{0}(\lambda)>0$ such that for every $R \geq R_{0}(\lambda)$, we have

$$
\left\|\tau_{\lambda} x\right\| \leq\|x\|, \quad \text { for } x \in \widetilde{\mathcal{K}} \text { with }\|x\|=R \text {. }
$$

Proof. Define $\tilde{f}(u)=\max _{0 \leq z \leq u} f(z)$. Clearly, $\tilde{f}$ is a nondecreasing function on $[0, \infty)$; moreover, since $f_{\infty}=0$, it is obvious that

$$
\lim _{u \rightarrow \infty} \frac{\tilde{f}(u)}{u}=0
$$

Therefore, we have that for $\varepsilon(\lambda)=1 / 2 \lambda M \int_{0}^{T} g(s) d s$, there exists $R_{1}(\lambda)>0$ such that $\tilde{f}(u) \leq \varepsilon u$ for each $u \geq R_{1}(\lambda)$.

Define $R_{0}(\lambda):=\max \left\{R_{1}(\lambda), 2 \gamma^{*}\right\}$, fix $R \geq R_{0}(\lambda)$, and let $x \in \widetilde{\mathcal{K}}$ with $\|x\|=R$. Then

$$
\begin{aligned}
\tau_{\curlywedge} x(t) & =\lambda \int_{0}^{T} G(t, s) g(s) f(x(s)) d s+\gamma(t) \\
& \leq \lambda \int_{0}^{T} G(t, s) g(s) \tilde{f}(\|x\|) d s+\gamma(t) \\
& \leq \lambda M \varepsilon\|x\| \int_{0}^{T} g(s) d s+\gamma^{*} \\
& =\frac{R}{2}+\gamma^{*} \leq \frac{R}{2}+\frac{R}{2}=R=\|x\|,
\end{aligned}
$$

and thus $\left\|\tau_{\lambda} x\right\| \leq\|x\|$.

Theorem 3.5. Assume $(H 0),(\widetilde{H 1}),(H 2),(\widetilde{H 3})$, and $(H 4)$. The following results hold:

(1) if $\int_{0}^{T} \gamma(s) d s>0$. then there exists $\lambda_{0}>0$ such that problem (1.2) has a nonnegative solution if $0<\lambda<\lambda_{0}$,

(2) if $\int_{0}^{T} \gamma(s) d s>0$ and $f_{\infty}=0$, then problem (1.2) has a nonnegative solution for every $\lambda>0$.

Proof. The first assertion is a direct consequence of Lemmas 3.2 and 3.3. The second part follows from Lemmas 3.3 and 3.4

Now, we will impose a strong condition on function $g$ by assuming that $g$ is strictly positive on the whole interval.

$\widetilde{(H 2)} g \in L^{1}([0, T]), g(t) \geq g_{*}>0$ for a.e. $t \in[0, T]$. 
Lemma 3.6. Assume that conditions $(H 0), \widetilde{(H 1)}, \widetilde{(H 2)}, \widetilde{(H 3)}$, and $(H 4)$ are satisfied. Then, if $f_{0}=\infty$, there exists $r_{0}(\lambda)>0$ such that for every $0<r \leq r_{0}(\lambda)$, we have

$$
\left\|\tau_{\lambda} x\right\| \geq\|x\|, \text { for } x \in \widetilde{\mathcal{K}} \text { with }\|x\|=r \text {. }
$$

Proof. Since $f_{0}=\infty$ for $L=L(\lambda)=T / \lambda \beta \widetilde{\sigma} g_{*}$, there exists $r_{0}(\lambda)>0$ such that $f(u) \geq L u$ for each $0 \leq u \leq r_{0}(\lambda)$.

Fix $0<r \leq r_{0}(\lambda)$, and let $x \in \widetilde{\mathcal{K}}$ with $\|x\|=r$. Then,

$$
\begin{aligned}
\left\|\tau_{\lambda} x\right\| \geq \frac{1}{T} \int_{0}^{T} \tau_{\curlywedge} x(t) d t & =\frac{\lambda}{T} \iint_{0}^{T} G(t, s) g(s) f(x(s)) d s d t+\frac{1}{T} \int_{0}^{T} \gamma(t) d t \\
& \geq \frac{\lambda}{T} \iint_{0}^{T} G(t, s) g(s) f(x(s)) d t d s \\
& \geq \frac{\lambda}{T} \beta g_{*} L \int_{0}^{T} x(s) d s \\
& \geq \frac{\lambda}{T} \beta g_{*} L \tilde{\sigma}\|x\| \\
& =\|x\|,
\end{aligned}
$$

and thus $\left\|\tau_{\lambda} x\right\| \geq\|x\|$.

Now, we are in a position to present the main result of this section.

Theorem 3.7. Suppose that conditions $(H 0),(\widetilde{H 1}),(\widetilde{H 2}),(\widetilde{H 3})$, and $(H 4)$ are fulfilled. The following assertions are satisfied:

(1) if $f_{0}=\infty$, then there exists $\lambda_{0}>0$ such that problem (1.2) has a nonnegative solution if $0<\lambda<\lambda_{0}$,

(2) if $f_{0}=\infty$ and $f_{\infty}=0$, then problem (1.2) has a nonnegative solution for every $\lambda>0$,

(3) if $f_{0}>0$ and $f_{\infty}>0$, then there exists $\lambda_{0}>0$ such that problem (1.2) has no nonnegative solutions if $\lambda>\lambda_{0}$.

Proof. The first assertion is a direct consequence of Lemmas 3.2 and 3.6. The second part follows from Lemmas 3.4 and 3.6.

To prove Part 3 , by using that $f_{0}>0$ and $f_{\infty}>0$, we know that there exists $L>0$ such that $f(u) \geq L u$ for all $u \geq 0$.

By defining

$$
\lambda_{0}:=\frac{T}{\beta \widetilde{\sigma} L g_{*}},
$$


we have that if there is any $\lambda>\lambda_{0}$ for which there exists a nonnegative solution $x$ of problem (1.2), then $x=\tau_{\lambda} x \in \mathcal{K}$. So, we arrive at the following contradiction:

$$
\begin{aligned}
\left\|\tau_{\lambda} x\right\| \geq \frac{1}{T} \int_{0}^{T} \tau_{\lambda} x(t) d t & =\frac{\lambda}{T} \iint_{0}^{T} G(t, s) g(s) f(x(s)) d s d t+\frac{1}{T} \int_{0}^{T} \gamma(t) d t \\
& \geq \frac{\lambda}{T} \iint_{0}^{T} G(t, s) g(s) f(x(s)) d t d s \\
& \geq \frac{\lambda}{T} \beta g_{*} L \int_{0}^{T} x(s) d s \\
& \geq \frac{\lambda}{T} \beta g_{*} L \tilde{\sigma}\|x\| \\
& >\|x\| .
\end{aligned}
$$

\subsection{Applications to Singular Equations}

Despite the fact that in the previous results we deal with regular functions, it is possible to apply some of them to the singular equation

$$
x^{\prime \prime}(t)+a(t) x(t)=\lambda g(t) f(x(t))+c(t), \quad x(0)=x(T), \quad x^{\prime}(0)=x^{\prime}(T),
$$

by means of a truncation technique.

To this end, we will consider a function $f$ that satisfies

(H5) $f:(0, \infty) \rightarrow(0, \infty)$ is a continuous function such that $f_{\infty}=0$.

Theorem 3.8. Assume that $\gamma_{*}>0$ and conditions ( $\left.\widetilde{H 1}\right),(H 2),(H 4)$, and (H5) hold.

Then, problem (3.18) has a positive solution for every $\lambda>0$.

Proof. Let $r=\gamma_{*}>0$, and define the function

$$
f_{r}(u)= \begin{cases}f(r), & \text { if } 0 \leq u<r \\ f(u), & \text { if } u \geq r .\end{cases}
$$

From (H5), it follows that $f_{r}$ satisfies condition $(\widetilde{H 3})$, and $\left(f_{r}\right)_{\infty}=0$. Moreover, $\gamma_{*}>0$ implies that $\int_{0}^{T} \gamma(s) d s>0$. As consequence, Theorem 3.5, Part 2, implies that the modified problem

$$
x^{\prime \prime}(t)+a(t) x(t)=\lg (t) f_{r}(x(t))+c(t), \quad x(0)=x(T), \quad x^{\prime}(0)=x^{\prime}(T)
$$

has a nonnegative solution $x_{r}$ for all $\lambda>0$. Such function is given by the expression

$$
x_{r}(t)=\lambda \int_{0}^{T} G(t, s) g(s) f_{r}\left(x_{r}(s)\right) d s+\gamma(t)
$$


The nonnegativeness of functions $G, g$ and $f_{r}$ implies that the solution $x_{r}(t) \geq \gamma_{*}=r$ for all $t \in[0, T]$. Therefore, $x_{r}$ is a positive solution of problem (3.18).

Remark 3.9. Theorem 3.8 is an alternative result to those obtained in $[15,16]$ by means of Schauder's fixed point theorem.

Example 3.10. Let us consider the repulsive singular differential equation

$$
x^{\prime \prime}+a(t) x=\lg (t)\left(x^{1 / 2}-\ln (x)\right)+c(t)
$$

Since $f(x)=x^{1 / 2}-\ln (x)$ satisfies (H5), we can apply Theorem 3.8 to obtain the following.

Corollary 3.11. Assume that $(\widetilde{H 1}),(H 2)$, and (H4) hold. If $\gamma_{*}>0$ then, (3.22) has a positive $T$-periodic solution for every $\lambda>0$.

\section{Acknowledgment}

This work was partially supported by FEDER and Ministerio de Educación y Ciencia, Spain, project no. MTM2010-15314.

\section{References}

[1] J. R. Graef, L. Kong, and H. Wang, "Existence, multiplicity, and dependence on a parameter for a periodic boundary value problem," Journal of Differential Equations, vol. 245, no. 5, pp. 1185-1197, 2008.

[2] A. Cabada and N. D. Dimitrov, "Multiplicity results for nonlinear periodic fourth order difference equations with parameter dependence and singularities," Journal of Mathematical Analysis and Applications, vol. 371, no. 2, pp. 518-533, 2010.

[3] J. R. Graef, L. Kong, and H. Wang, "A periodic boundary value problem with vanishing Green's function," Applied Mathematics Letters, vol. 21, no. 2, pp. 176-180, 2008.

[4] D. Jiang, J. Chu, and M. Zhang, "Multiplicity of positive periodic solutions to superlinear repulsive singular equations," Journal of Differential Equations, vol. 211, no. 2, pp. 282-302, 2005.

[5] P. J. Torres, "Existence of one-signed periodic solutions of some second-order differential equations via a Krasnoselskii fixed point theorem," Journal of Differential Equations, vol. 190, no. 2, pp. 643-662, 2003.

[6] R. Ma, "Nonlinear periodic boundary value problems with sign-changing Green's function," Nonlinear Analysis, Theory, Methods and Applications, vol. 74, no. 5, pp. 1714-1720, 2011.

[7] P. J. Torres and M. Zhang, "A monotone iterative scheme for a nonlinear second order equation based on a generalized anti-maximum principle," Mathematische Nachrichten, vol. 251, pp. 101-107, 2003.

[8] A. Cabada and J. Á. Cid, “On the sign of the Green's function associated to Hill's equation with an indefinite potential," Applied Mathematics and Computation, vol. 205, no. 1, pp. 303-308, 2008.

[9] A. Cabada, J. Á. Cid, and M. Tvrdý, "A generalized anti-maximum principle for the periodic one-dimensional $p$-Laplacian with sign-changing potential," Nonlinear Analysis. Theory, Methods $\mathcal{E}$ Applications, vol. 72, no. 7-8, pp. 3436-3446, 2010.

[10] M. Zhang, "A relationship between the periodic and the Dirichlet BVPs of singular differential equations," Proceedings of the Royal Society of Edinburgh. Section A, vol. 128, no. 5, pp. 1099-1114, 1998.

[11] M. Zhang, “Optimal conditions for maximum and antimaximum principles of the periodic solution problem," Boundary Value Problems, vol. 2010, Article ID 410986, 26 pages, 2010.

[12] M. A. Krasnoselskii, Positive Solutions of Operator Equations, P. Noordhoff Ltd., Groningen, The Netherlands, 1964. 
[13] E. Esmailzadeh and G. Nakhaie-Jazar, "Periodic solution of a Mathieu-Duffing type equation," International Journal of Non-Linear Mechanics, vol. 32, no. 5, pp. 905-912, 1997.

[14] V. Bevc, J. L. Palmer, and C. Süsskind, "On the design of the transition region of axi-symmetric magnetically focusing beam valves," British Institution of Radio Engineers, vol. 18, pp. 696-708, 1958.

[15] J. Chu and Z. Zhang, "Periodic solutions of singular differential equations with sign-changing potential," Bulletin of the Australian Mathematical Society, vol. 82, no. 3, pp. 437-445, 2010.

[16] P. J. Torres, "Weak singularities may help periodic solutions to exist," Journal of Differential Equations, vol. 232, no. 1, pp. 277-284, 2007. 


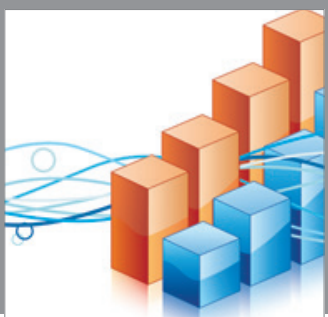

Advances in

Operations Research

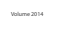

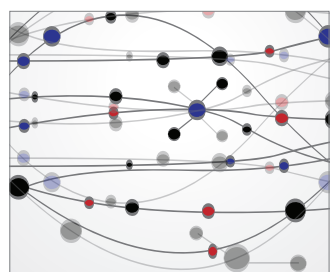

\section{The Scientific} World Journal
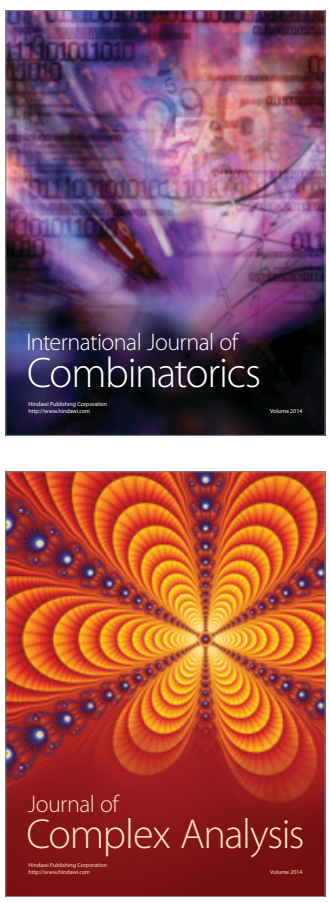

International Journal of

Mathematics and

Mathematical

Sciences
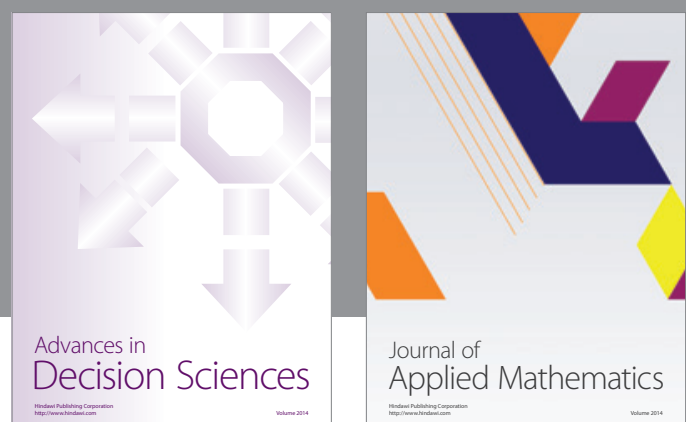

Journal of

Applied Mathematics
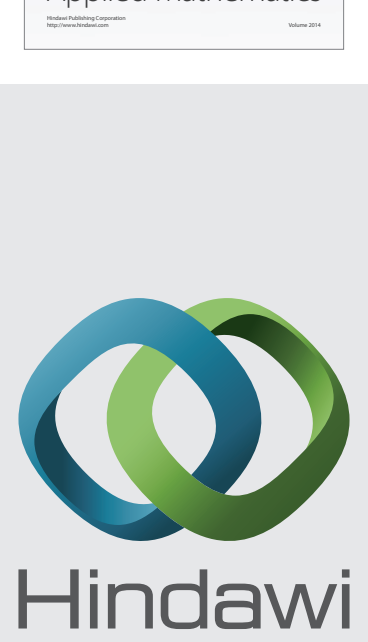

Submit your manuscripts at http://www.hindawi.com
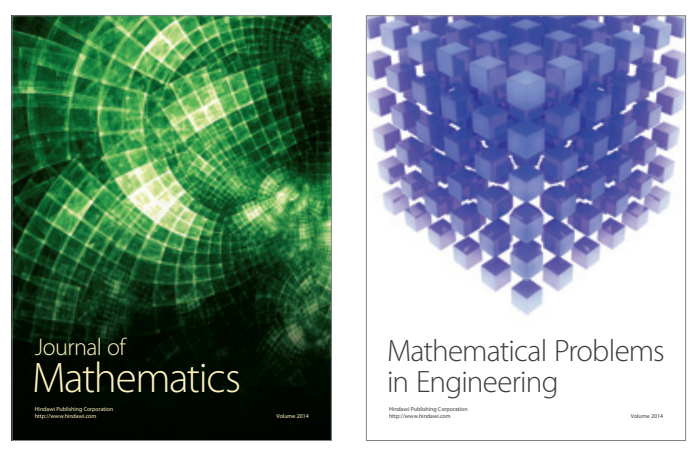

Mathematical Problems in Engineering
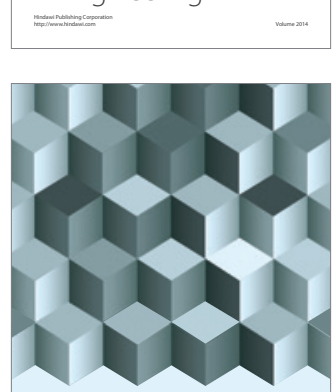

Journal of

Function Spaces
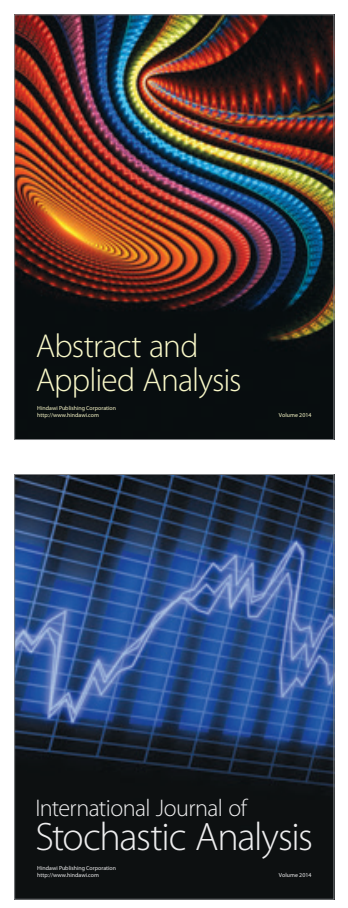

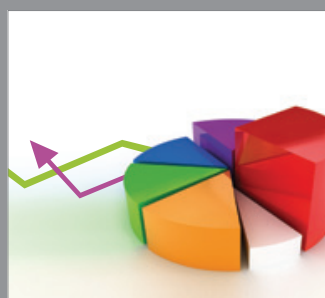

ournal of

Probability and Statistics

Promensencen
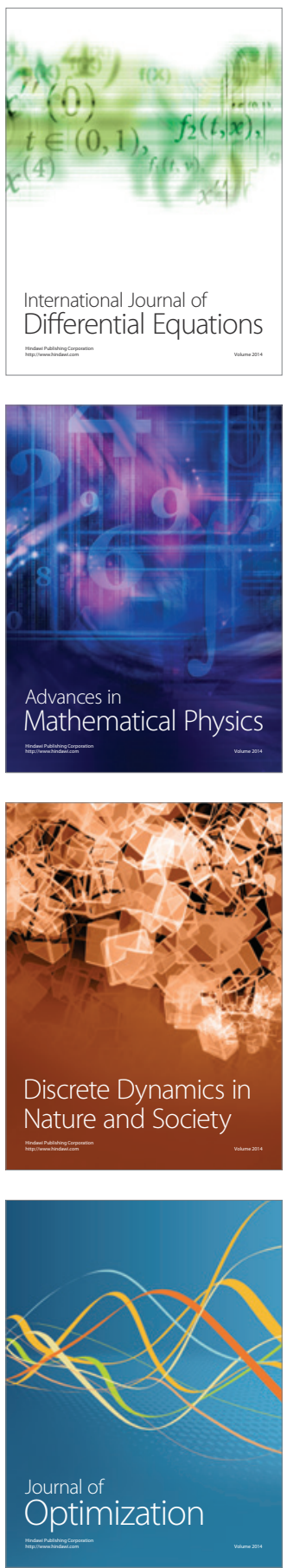